\title{
On a Possibly Pure Set-Theoretic Contribution to Black Hole Entropy
}

\author{
Gábor Etesi ${ }^{1}$ (D) \\ Published online: 19 September 2019 \\ (c) The Author(s) 2019
}

\begin{abstract}
Continuity as appears to us immediately by intuition (in the flow of time and in motion) differs from its current formalization, the arithmetical continuum or equivalently the set of real numbers used in modern mathematical analysis. Motivated by the known mathematical and physical problems arising from this formalization of the continuum, our aim in this paper is twofold. Firstly, by interpreting Chaitin's variant of Gödel's first incompleteness theorem as an inherent uncertainty or fuzziness of the arithmetical continuum, a formal set-theoretic entropy is assigned to the arithmetical continuum. Secondly, by analyzing Noether's theorem on symmetries and conserved quantities, we argue that whenever the four dimensional space-time continuum containing a single, stationary, asymptotically flat black hole is modeled by the arithmetical continuum in the mathematical formulation of general relativity, the hidden set-theoretic entropy of this latter structure reveals itself as the entropy of the black hole (proportional to the area of its "instantaneous" event horizon), indicating that this apparently physical quantity might have a pure set-theoretic origin, too.
\end{abstract}

Keywords Continuum · Chaitin incompleteness · Noether theorem · Black hole entropy

\section{Introduction}

In his struggling with Zeno's paradoxes introduced into the Western thinking in an apparently never-more-removable way by the Eleatic school, Aristotle declared that even by performing an infinitude of successive divisions the extension of time or space cannot be dissolved or destroyed (this is one particular form of Aristotle's general prohibition of the appearance of actual infinities). In other words the Aristotelian description of the continuum considered the (temporal or spatial) extension as a primitive, irreducible concept. This Aristotelian or let us call synthetical concept of the continuum is however in sharp contrast with our contemporary analytical approach to the continuum used in both mathematics and theoretical physics. Accepting and making good use of the concept of the analytical continuum when solving mathematical or physical problems

Gábor Etesi

etesi@math.bme.hu

1 Department of Geometry, Mathematical Institute, Faculty of Science, Budapest University of Technology and Economics, Egry J. u. 1, H ép., Budapest 1111, Hungary 
means that one declares that the ultimate constituents of the (temporal or spatial) continuum are the points without possessing any extension and the analytical continuum with its all pleasant properties arises as the disjoint union of its ultimate constituents, the extensionless points.

Taking a rapid overview of its history, the synthetical approach to the continuum seems to had been accepted after Aristotle till Descartes (recall his dualistic philosophy based on the res extensa and the res cogitans). However in a sudden sharp transition, the next generation, hallmarked by such names as Leibniz and Newton, began to replace the synthetical description with the analytical one and this new but intuitive approach was finally strictly formalized by Dedekind in 1858. Concerning the character and a possible root of this transition let us quote from Russell (1910, Chapter IX):

Even among philosophers, we may say, broadly, that only those universals which are named by adjectives or substantives have been much or often recognized, while those named by verbs and prepositions have been usually overlooked. This omission has had a very great effect upon philosophy; it is hardly too much to say that most metaphysics, since Spinoza, has been largely determined by it. The way this has occured is, in outline, as follows: Speaking generally, adjectives and common nouns express qualities or properties of single things, whereas prepositions and verbs tend to express relations between two or more things. Thus the neglect of prepositions and verbs led to the belief that every proposition can be regarded as attributing a property to a single thing, rather than as expressing a relation between two or more things. Hence it was supposed that, ultimately, there can be no such entities as relations between things. Hence either there can be only one thing in the universe, or, if there are more than one things, they cannot possibly interact in any way, since any interaction would be a relation, and relations are impossible.

The first of these views, advocated to Spinoza and held in our own day by Bradley and many other philosophers, is called monism; the second, advocated to Leibniz but not very common nowadays, is called monadism, because each of the isolated things is called a monad. Both these opposing philosophies, interesting as they are, result, in my opinion, from an undue attention to one sort of universals, namely the sort represented by adjectives and substantives rather than by verbs and prepositions.

It therefore seems that the passage from the synthetical to the analytical approach at least in part was a consequence of the slow decline of the influensive and in the beginning deep and from the conceptional viewpoint colourful universalia-realia debate of the previous scholastic centuries hence it was not so sharp. Nevertheless the central figure of this conceptual shift was surely Leibniz who played an important role in the developments of both seventeenth-eighteenth century philosophy with his monadism and mathematics with his fundamental contributions to mathematical analysis. Indeed, there is a more or less straightforward analogy between the purely metaphysical concept of a monad and the formal mathematical concept of a(n extensionless) point: Both philosophically and mathematically speaking, they represent sort of irreducible terminal elements of reality, nonetheless possessing the character of totality in the sense that they are the only constituents of existing things, moreover they are disjoint entities without interacting with each other.

The great success of modern mathematical analysis and theoretical physics based on the utilization of the analytical continuum is well-known and cannot be overestimated; however the present-day analytical formalization of continuity, which dissolves it into the collection of extensionless points, is not obvious intuitively at all since its acceptance in mathematics. As Weyl calls attention (Weyl 1994, pp. 23-24): 
But as things now stand we must point out that, in spite of Dedekind, Cantor, and Weierstrass, the great task which has been facing us since the Pythagorean discovery of the irrationals remains today as unfinished as ever; that is, the continuity given to us immediately by intuition (in the flow of time and in motion) has yet to be grasped mathematically as a totality of discrete "stages" in accordance with that part of its content which can be conceptualized in an "exact" way. More or less arbitrarily axiomatized systems (be they ever so "elegant" and "fruitful") cannot further help us here. We must try to attain a solution which is based on objective insight.

Still quoting Weyl (1994, p. 49):

If, as I have advocated, we give a precise meaning to the concept "set", then the following assertion gains a substantial content: "To every point of a line (given an origin and a unit of length) there corresponds a (distance-measuring) real number [...] and vice versa". This assertion establishes a noteworthy connection between something given in the intuition of space and something constructed in a logical conceptual way. But, clearly, this assertion far exceeds everything which intuition teaches, or can teach, us about the continuum. For it does not offer a morphological description of what presents itself in intuition (that being, first and foremost, a fluid whole rather than a set of discrete elements). Instead, it gives an exact construal of an immediately given reality which by its nature, is inexact - a process which is fundamental to all exact knowledge of (physical) reality and through which alone mathematics acquires significance for natural science.

These worries are not of pure academic nature taking into account that the concept of the analytical continuum, or as we shall call in its currently formalized form here from now on, the arithmetical continuum (or equivalently the set $\mathbb{R}$ of real numbers), is widely used everywhere in mathematics, physics, engineering sciences, mathematical biology, economy and sociology, etc., etc. Indeed, in spite of its success, the utilization of the arithmetical continuum leads to various problems in pure mathematics (Weyl 1994) and in its applications, too (for an excellent survey see Baez 2016). Perhaps the most relevant problem related with the arithmetical continuum on the pure mathematical side is the (in our opinion) unsettled status of Cantor's continuum hypothesis; while a simple example from theoretical physics is the divergence of the total electric energy of an electrically charged point particle in Faraday-Maxwell electrodynamics (leading eventually to the complicated renormalization issues in classical and quantum field theories). Fortunately, by performing simple experiments, we know that this and the various other occurences of divergences in classical and quantum electrodynamics are nothing but artifacts originating from the mathematical formulation (which uses the arithmetical continuum) of these theories hence we can identify and isolate these divergences quite easily. But what about the singularities or other phenomena (mathematically) predicted by general relativity for instance? Lacking unambigous experimental evidences yet we cannot make a commitment about their ontological status.

The purpose of this paper is to examine whether the several conceptional, technical, etc. problems related with black hole entropy (Bardeen et al. 1973) at least in part emanate from the tacit fact that mathematically general relativity rests on the structure of the arithmetical continuum. The paper is organized as follows. In Sect. 2 we recall Chaitin's reformulation of Gödel's first incompleteness theorem and interpret its content as the presence of an inherent uncertainty or fuzziness within the arithmetical continuum (which might be a consequence that its final constituents are extensionless). This allows us to introduce a 
statistico-physical analogy and to talk in this context about the pure "set-theoretic entropy" of the arithmetical conntinuum. Then, in Sect. 3 we recall and refine Noether's theorem on symmetries and conserved quantities and prove that within general relativity a conserved quantity to diffeomorphisms can be assigned which is not zero if a stationary black hole is present and can be identified with its entropy (proportional to the area of the "instantaneous" event horizon, Hawking 1971). Then we argue that, based on their common diffeomorphism invariance, the "set-theoretic" and the black hole entropy are in fact the same. This suggests that the latter entropy might be a consequence of the former one and the long-sought physical degrees of freedom responsible for black hole entropy have simply a pure mathematical origin corresponding to the artificial decomposition of the space-time continuum into points.

\section{Chaitin Incompleteness and the Entropy of the Continuum}

Accepting the structure of the arithmetical continuum or equivalently the set $\mathbb{R}$ of real numbers, ${ }^{1}$ it is the totality of, or more precisely the disjoint union of, its individual constituents called real numbers or-speaking geometrically—its points:

$$
\mathbb{R}=\bigsqcup_{x \in \mathbb{R}}\{x\} .
$$

If indeed this is its optimal mathematical structure, then one would expect that using mathematical tools only, one is able to "locate" the individual constituents $x$ within their totality $\mathbb{R}$ or equivalently, one is able to make a mathematical distinction between them in an effective way. We know from our elementary university studies that upon fixing a notational convention every real number admits a well-defined (for instance decimal) expansion which means that this expansion exists for all real numbers and is unique in the sense that two expansions coincide if and only if the corresponding two real numbers are equal. It is however a quite surprising observation that in general the existence of this well-defined and unique expansion is the only available property of a "truely generic" real number i.e. a typical element $x \in \mathbb{R}$. Therefore our question about an effective mathematical way of "picking" a single element $x \in \mathbb{R}$ is in fact a question about the effectiveness of making distinctions between generic real numbers in terms of their (decimal) expansions.

To approach this problem first let us recall the idea of Kolmogorov complexity or algorithmic compressibility or computability of a real number. Let $x \in \mathbb{R}$ be given and denote by $\mathbf{T}_{x}$ the (probably empty) set of those Turing machines which reproduce $x$ in the sense that if $\mathbf{T}_{x} \neq \emptyset$ and some $T_{x} \in \mathbf{T}_{x}$ is given with an input $n \in \mathbb{N}$ then the output $T_{x}(n) \in \mathbb{N}$ consists of e.g. precisely the first $n$ digits of the expansion of $x$. Denoting by $\left|T_{x}\right| \in \mathbb{N}$ the length of the Turing machine (considered as an algorithm or program in some programming language) define

$$
K(x):= \begin{cases}+\infty & \text { if } \mathbf{T}_{x}=\emptyset \\ \inf _{T_{x} \in \mathbf{T}_{x}}\left|T_{x}\right|<+\infty & \text { if } \mathbf{T}_{x} \neq \emptyset\end{cases}
$$

\footnotetext{
${ }^{1}$ Here by a real number $x$ we mean by definition a Dedekind slice and by the set of real numbers $\mathbb{R}$ the set of all Dedekind slices. $\mathbb{R}$ can be equipped with the usual structures (addition, multiplication and ordering) and posssesses the completeness property rendering it a complete ordered field. It is well-known that $\mathbb{R}$ as a complete ordered field is essentially unique.
} 
and call the resulting (extended) natural number $K(x) \in \mathbb{N} \cup\{+\infty\}$ the Kolmogorov complexity of $x \in \mathbb{R}$. Then $K(x)=+\infty$ corresponds to the situation when no algorithms reproducing $x$ in the above sense exist hence $x$ is not algorithmically compressible or not computable (by simple cardinality arguments the vast majority of real numbers belongs to this class) while $K(x)<+\infty$ corresponds to the opposite case (this class contains all "known" real numbers like $\left.7, \frac{5}{8}, \sqrt{2}, \pi, \mathrm{e}, \ldots\right)$. It is clear that the only important question about $x$ in this context is whether $K(x)=+\infty$ or $K(x)<+\infty$ and in the latter case only the magnitude of $K(x)$ is relevant, for its particular value depends on the details of the sort of expansion of $x$, the programming language for $T_{x}$, etc. hence does not carry essential information. A remarkable observation about Kolmogorov complexity is the following result (Chaitin 1992):

Theorem 2.1 (Chaitin's version of Gödel's first incompleteness theorem) For any (sufficiently rich, consistent, recursively enumerable) axiomatic system $S$ based on a first order language $L$ there exists a natural number $0<N_{S}<+\infty$ such that there exists no real number $x$ for which the proposition

$$
K(x) \geqq N_{S}
$$

is provable within $S$.

Motivated in various ways by Geroch and Hartle (1986) and Schlesinger (2014a, b) we interpret this quite surprising mathematical fact from our viewpoint as follows: taking into account that the only known property of a generic real number which fully characterizes it is its existing (decimal) expansion, but the Kolmogorov complexity of this expansion hence the expansion itself generally is not fully determinable (by proving theorems on it in an axiomatic system), there is in general no way, using standard mathematical tools in the broadest sense, to "sharply pick" any element from the arithmetical continuum. Consequently, from the viewpoint of "effective mathematical activity", the structure of the arithmetical continuum i.e. the set $\mathbb{R}$ of real numbers contains an inherent uncertainty or fuzziness in the sense that its individual disjoint constituents cannot be distinguished from each other in a universal and effective mathematical way.

The above interpretation of Theorem 2.1 serves as a motivation to introduce a statisticophysical analogy for the arithmetical continuum which might offer another insight into its structure. First, generalizing the above decomposition of the real line into its points, we suppose that every (finite dimensional, real) differentiable manifold $M$ admits a decomposition into its disjoint constituent points:

$$
M=\bigsqcup_{x \in M}\{x\} .
$$

Speaking intutively, we can make three observations about this decomposition: All the points $x$ of $M$ (i) are homologous i.e. "look the same", (ii) are terminal objects i.e. they do not possess any further internal structure nevertheless their collection gives back precisely $M$ and (iii) are disjoint from each other i.e. they "do not interact". Except its cardinality this decomposition of $M$ therefore strongly resembles the structure of an ideal gas as usually defined in statistical physics. Take an abstract set $X$ whose cardinality coincides with that of the continuum in ZFC set theory and regard it as an abstract ideal gas $X$ such that its elements $x$ correspond to the atoms $x$ of the ideal gas $X$. Extending this analogy further, the left hand side of (1) i.e. the fixed diffeomorphism class of $M$ with its global topological, 
smooth, etc. "macroscopic" properties can be regarded as one possible macrostate of $X$ while the right hand side of (1) i.e. the particular identification of $M$ with its elements as a particular microstate of $X$ within the macrostate $M$. The dynamics of $X$ in its macrostate $M$ is generated by diffeomorphisms; hence another microstate within the same macrostate is achieved by picking any diffeomorphism $f: M \rightarrow M$ and taking the corresponding new decomposition

$$
M=f(M)=\bigsqcup_{x \in M}\{f(x)\} .
$$

Another differentiable manifold $N$ not diffeomorphic to $M$ (in the broadest sense i.e. possibly having different dimension, number of connected components, etc.) might be interpreted as a different macrostate (with its corresponding assembly of microstates created by diffeomorphisms) of the same astractly given ideal gas $X$. However this abstract ideal gas can even appear in completely different i.e. non-geometric, discontinuous macrostates as well like e.g. in the form of the Cantor set $C \subset \mathbb{R}$ or some other abstract topological space (with its homeomorphisms creating the corresponding assembly of microstates), or just simply in the form of some set (together with its bijections), etc., etc.

In accord with this analogy Theorem 2.1 is interpreted as a fundamental result about the indistinguishability of the individual microstates of $X$ belonging to the same macrostate $M$. The next standard step in statistical mechanics is then to introduce a tool, a measure, capable to grasp the amount of information loss created by the passage from individual microstates to their common macrostate. This measure is known as the entropy of the ideal gas in a given macrostate. How could we characterize this entropy within our analogy? Proceeding completely formally along the way of Boltzmann's classical approach to entropy we can argue as follows. Certainly all possible microstates of the abstract ideal gas $X$ are parameterized by the elements of the group of its all set-theoretic bijections $\operatorname{Bij}(X)$ while its possible microstates within the macrostate $M$ are parameterized by its subgroup of diffeomorphisms $\operatorname{Diff}(M)$. Thus we formally put

Entropy of the set $X$ in its manifold-macrostate $M:=$ Const $_{S} \cdot \log \Gamma_{S}(\operatorname{Diff}(M))$

with $\Gamma_{S}$ being an, at this state of the art admittedly hypothetical, measure on $\operatorname{Bij}(X)$ depending on a particular choice of axiomatic system $S$ in Theorem 2.1. Taking into account $1 \varsubsetneqq \operatorname{Diff}(M) \varsubsetneqq \operatorname{Bij}(X)$ we expect $1<\Gamma_{S}(\operatorname{Diff}(M))<\Gamma_{S}(\operatorname{Bij}(X))=+\infty$ to hold such that, together with $0<$ Const $_{S}<+\infty$ the resulting formal expression is a finite positive number independent of any choice of $S$ as dictated by the universality of Theorem 2.1. Note that despite being formally ill-defined, by construction this entropy formula is invariant under diffeomorphisms of $M$.

To summarize, we have sketched a framework in which the inherent uncertainty or fuzziness of the arithmetical continuum i.e. the set $\mathbb{R}$ of real numbers or gemetrically speaking the real line or more generally any differentiable manifold, can be interpreted as a non-zero entropy of the arithmetical continuum (cf. Schlesinger 2014a, b).

\section{Secondary Noether Theory and the Entropy of Black Holes}

We would like to make the quite nonsense formula (2) more meaningful by passing from mathematics to physics. Namely, we shall consider classical physical theories over physical space-time such that in the mathematical description of these theories the physical 
space-time is modeled on a differentiable manifold with the property (1). Then, we shall ask ourselves: Does the inherent uncertainty or fuzziness of the arithmetical continuum recognized in the mathematical model of the physical theory "pop up" somehow among the physical propositions of the physical theory? Putting differently: Does this fuzziness somehow "lift" from the mathematical level to the physical level of the physical theory? Since we have found some similarities between this purely mathematical uncertainty or fuzziness of the arithmetical continuum and the physical concept of entropy, we are going to seek entropylike phenomena in those physical theories which are particularly sensitive for the physical structure of space-time. If these sought entropylike phenomena happen to have a pure set-theoretical origin introduced by the mathematical description of the physical theory, then we expect them to have something to do with diffeomorphisms of the underlying differentiable manifold modeling physical space-time; for the formal expression (2) is diffeomorphism-invariant hence the entropy it describes is invariant under diffeomorphisms. Apart from this, if diffeomorphisms are in addition symmetries of the physical theory we are dealing with then we may as well try to identify these entropylike things with Noether charges associated with diffeomorphism symmetry.

By Noether's theorem in a broad physical sense one means that "to every continuous symmetry of a physical theory a quantity can be assigned which is conserved". It may happen however that this conserved quantity, the Noether charge, vanishes. Our goal is to demonstrate that even in this trivial case certain non-trivial de Rham cohomology classes can still be interpreted as "secondary Noether charges" associated with this symmetry of the theory. There is an analogouos situation in algebraic topology. Consider a complex vector bundle $E$ over a topological space $X$. Recall that for all $i=0, \ldots$, rk $E$ the $i$ th Chern class of $E$ takes value in $H^{2 i}(X ; \mathbb{Z})$. Therefore, if it happens that $X$ has vanishing even dimensional singular cohomology then characteristic classes cannot be used to distinguish complex vector bundles over it. ${ }^{2}$ However if $X$ is a manifold $M$ then one can still introduce the socalled secondary or Chern-Simons characteristic classes taking values in odd dimensional cohomology (Chern and Simons 1974). Motivated by this consideration as well as those in Dolan (2018) and Wald (1993) we proceed as follows.

For completeness and convenience let us recall how standard Noether theory works in case of a classical relativistic field theory. We are going to skip all the technical details here but emphasize that in case of a compact, orientable Riemannian 4-manifold all of our considerations below are rigorous mathematical statements; therefore we have a reason to hope that with appropriate technical modifications all the stuff remains valid in physically more realistic situations.

So let $(M, g)$ be a four dimensional (non-)compact oriented (pseudo-)Riemannian manifold representing space-time and let $\Phi$ denote the full field content of a classical field theory over $(M, g)$ defined by a Lagrangian density $L(\Phi, g) \in \Omega^{4}(M)$. Note that by definition the Lagrangian is not a function but a 4-form over $M$ allowing one to talk about the corresponding action $S(\Phi, g)=\int_{M} L(\Phi, g)$ defined by integration over $M$. Let $\mathscr{M}$ be the configuration space of all (but belonging to a nice function class) $(\Phi, g$ )-field configurations over $M$ i.e. its elements are not identified by diffeomorphisms, gauge, etc. transformations.

\footnotetext{
2 A simple example for this failure is provided by complex rank two vector bundles with structure group SU(2) over the 5-sphere $S^{5}$. Then, on the one hand, isomorphism classes of these type of vector bundles are classified by the group $\pi_{4}(\mathrm{SU}(2)) \cong \pi_{4}\left(S^{3}\right) \cong \mathbb{Z}_{2}$ hence there are precisely two different such bundles up to isomorphism over $S^{5}$; meanwhile, on the other hand, $H^{k}\left(S^{5} ; \mathbb{Z}\right) \cong\{0\}$ if $k \neq 0,5$ hence all Chern classes of these bundles are trivial.
} 
Consider a differentiable curve $C: \mathbb{R} \rightarrow \mathscr{M}$. We say that $C$ is a symmetry of the theory $L(\Phi, g)$ if its action $S(\Phi, g)$ is constant along $C$ that is, $S(C(t))=S(\Phi(t), g(t))=$ Const for all $t \in \mathbb{R}$. Writing $(\Phi, g):=(\Phi(0), g(0))$ and using physicists' usual notation define "the infinitesimal variation of the action at $(\Phi, g)$ along $C$ ' by

$$
\delta_{C} S(\Phi, g):=\lim _{t \rightarrow 0} \frac{1}{t}(S(C(t))-S(C(0)))=\int_{M} \lim _{t \rightarrow 0} \frac{1}{t}(L(C(t))-L(C(0)))=: \int_{M} \delta_{C} L(\Phi, g)
$$

where $\delta_{C} L(\Phi, g) \in \Omega^{4}(M)$ is the "infinitesimal variation of the Lagrangian at $(\Phi, g)$ along $C^{\prime}{ }^{3}$ Assume that $(M, g)$ is smooth and let $\Delta_{g}:=\mathrm{dd}^{*}+\mathrm{d}^{*} \mathrm{~d}$ denote its Hodge Laplacian; recall that if $\omega \in \Omega^{4}(M)$ the partial differential equation $\Delta_{g} \varphi=\omega$ has a smooth solution $\varphi$ if and only if $\int_{M} \omega=0$. By definition of a symmetry $\int_{M} \delta_{C} L(\Phi, g)=0$ hence there exists an element $\varphi_{C} \in \Omega^{4}(M)$ satisfying $\Delta_{g} \varphi_{C}=\delta_{C} L(\Phi, g)$. However $\Delta_{g} \varphi_{C}=\mathrm{dd}^{*} \varphi_{C}+\mathrm{d}^{*} \mathrm{~d} \varphi_{C}=\mathrm{dd}^{*} \varphi_{C}$ consequently picking an arbitrary $\eta_{C} \in \Omega^{2}(M)$ and putting

$$
\theta_{C}:=\mathrm{d}^{*} \varphi_{C}+\mathrm{d} \eta_{C}
$$

we succeeded to find an element $\theta_{C} \in \Omega^{3}(M)$ such that $\mathrm{d} \theta_{C}=\delta_{C} L(\Phi, g)$. Note first that, although $\varphi_{C}$ is well-defined only up to a harmonic 4-form i.e. an element $\varphi \in \operatorname{Ker} \Delta_{g}$, the 3 -form $\theta_{C}$ is not sensitive for this ambiguity because taking into account its harmonicity, $\varphi$ is both closed $(\mathrm{d} \varphi=0)$ and co-closed $\left(\mathrm{d}^{*} \varphi=0\right)$ hence $\theta_{C}=\mathrm{d}^{*} \varphi_{C}+\mathrm{d} \eta_{C}=\mathrm{d}^{*}\left(\varphi_{C}+\varphi\right)+\mathrm{d} \eta_{C}$. Secondly, the "gauge freedom" i.e. the $\eta_{C}$-ambiguity can be fixed as well by imposing the "Coulomb gauge condition" $\mathrm{d}^{*} \theta_{C}=0$. Indeed, $\mathrm{d}^{*} \theta_{C}=\mathrm{d}^{* 2} \varphi_{C}+\mathrm{d}^{*} \mathrm{~d} \eta_{C}=\mathrm{d}^{*} \mathrm{~d} \eta_{C}=0$ (together with the Hodge decomposition theorem) implies $\mathrm{d} \eta_{C}=0$. Therefore, given a symmetry $C$ of the theory we come up with a $\theta_{C} \in \Omega^{3}(M)$ which satisfies

$$
\left\{\begin{array}{l}
\mathrm{d} \theta_{C}=\delta_{C} L(\Phi, g) \\
\mathrm{d}^{*} \theta_{C}=0 \\
\int_{M} \mathrm{~d} \theta_{C}=0
\end{array}\right.
$$

and this 3-form is well-defined in the sense that it depends only on the symmetry represented by the curve $C$ as expected. ${ }^{4}$

Proceeding further, we call the Hodge dual 1-form $j_{C}:=* \theta_{C} \in \Omega^{1}(M)$ the Noether current associated with the symmery $C$ moreover for a (spacelike) hypersurface-withoutboundary $S \subset M$ put $q_{C, S}:=\int_{S} * j_{C}$ and call it the Noether charge associated with the symmetry $C$. The Noether charge satisfies

$$
q_{C, S_{1}}-q_{C, S_{2}}= \pm \int_{W\left(S_{1}, S_{2}\right)} \mathrm{d} \theta_{C}=0
$$

\footnotetext{
${ }^{3}$ Using standard notations of differential geometry if $\dot{C}$ is the derivative of $C$ at $t=0$ then $\delta_{C} L=\dot{C}(L): \mathscr{M} \rightarrow \Omega^{4}(M)$. By specializing the variation further we can demand $\delta^{2}=0$ hence we can formally treat $\delta$ as an exterior derivative on the infinite dimensional manifold $\mathscr{M}$ (Dolan 2018, and references therein) and can introduce the $\Omega^{4}(M)$-valued 1-form $\delta L$ on $\mathscr{M}$. Then $\delta_{C} L=\delta L(\dot{C}): \mathscr{M} \rightarrow \Omega^{4}(M)$ as well.

${ }^{4}$ If $\left(\Phi_{0}, g_{0}\right) \in \mathscr{M}$ is a critical point of the action then $\delta_{C} S\left(\Phi_{0}, g_{0}\right)=\int_{M} \delta_{C} L\left(\Phi_{0}, g_{0}\right)=\int_{M} \delta L\left(\Phi_{0}, g_{0}\right)$ $(\dot{C})=0$ for all curves passing through the critical point. Hence $\int_{M} \delta L\left(\Phi_{0}, g_{0}\right)(\dot{C})=0$ for all $\dot{C} \neq 0$ thus in fact $\delta L\left(\Phi_{0}, g_{0}\right)=0$ which is the resulting field equation of the theory.
} 
by applying Stokes' theorem on a domain $W\left(S_{1}, S_{2}\right) \subseteq M$ with induced orientation and oriented boundary $\partial W\left(S_{1}, S_{2}\right)=S_{1} \sqcup\left(-S_{2}\right)$. (Here we strictly speaking assume that the variation vanishes on the complement $M \backslash W\left(S_{1}, S_{2}\right)$ hence $\int_{W\left(S_{1}, S_{2}\right)} \mathrm{d} \theta_{C}=\int_{M} \mathrm{~d} \theta_{C}=0$ indeed.) Consequently the real number $q_{C}:=q_{C, S}$ is a well-defined conserved quantity associated with the symmetry of the theory in this sense.

Thanks to the gauge fixing condition $\mathrm{d}^{*} \theta_{C}=0$ the Noether current looks like $j_{C}=* \theta_{C}= \pm \mathrm{d} * \varphi_{C}$ consequently $j_{C} \in[0] \in H^{1}(M)$ i.e. the current represents the trivial cohomology class in the first de Rham cohomology. We may then ask ourselves what about $\theta_{C}$ from the de Rham theoretic viewpoint? Does it represent a cohomology class in $H^{3}(M)$ ? Still working in the gauge $\mathrm{d}^{*} \theta_{C}=0$, assume $\mathrm{d} \theta_{C}=0$ holds; then via (3) we get $\Delta_{g} \varphi_{C}=0$ implying $\varphi_{C}$ is harmonic hence $\theta_{C}=\mathrm{d}^{*} \varphi_{C}=0$. Therefore we find that $\mathrm{d} \theta_{C}=0$ if and only if $\theta_{C}=0$. Consequently $\theta_{C} \in \Omega^{3}(M)$ represents a cohomology class $\left[\theta_{C}\right] \in H^{3}(M)$ if and only if $\theta_{C}=0$ and the associated Noether charge $q_{C}=\int_{S} * j_{C}= \pm \int_{S} \theta_{C}=0$ is trivial rendering the classical Noether theory useless in this situation.

Let us focus attention to this trivial case i.e. when for a symmetry $C$ of the theory $L(\Phi, g)$ the associated total derivative satisfies $\left[\theta_{C}\right] \in H^{3}(M)$ or equivalently (by exploiting the gauge fixing condition $\left.\mathrm{d}^{*} \theta_{C}=0\right) \theta_{C}=0$. This latter equation also implies $\mathrm{d}^{*} \varphi_{C}=0$ as we have seen hence the general expression (3) reduces to

$$
0=\mathrm{d} \eta_{C}
$$

saying that $\eta_{C}$ itself represents a cohomology class in $H^{2}(M)$. Consequently in this situation-which is trivial from the variational viewpoint in the sense that it yields vanishing primary Noether theory, but not trivial from the topological viewpoint in the sense that $H^{2}(M) \not\{0\}$ may hold—we can still introduce a secondary or topological Noether current $J_{C} \in \Omega^{2}(M)$ by putting $J_{C}:=* \eta_{C}$. Then taking any (spacelike) two dimensional submanifold-without-boundary $\Sigma \subset M$ the corresponding secondary or topological Noether charge $Q_{C, \Sigma}:=\int_{\Sigma} * J_{C}$ is well-defined in the sense that it depends only on the chosen de Rham cohomology class $\left[* J_{C}\right] \in H^{2}(M)$. Moreover

$$
Q_{C, \Sigma_{1}}-Q_{C, \Sigma_{2}}= \pm \int_{W\left(\Sigma_{1}, \Sigma_{2}\right)} \mathrm{d} \eta_{C}=0
$$

by Stokes' theorem. (This time $W\left(\Sigma_{1}, \Sigma_{2}\right) \subset M$ is a sub-3-manifold with induced orientation and oriented boundary $\partial W\left(\Sigma_{1}, \Sigma_{2}\right)=\Sigma_{1} \sqcup\left(-\Sigma_{2}\right)$.) Consequently we have a conserved quantity in the sense that the number $Q_{C, \Sigma}=: Q_{C,[\Sigma]}$ depends only on $\left[* J_{C}\right] \in H^{2}(M)$ and the singular homology class $[\Sigma] \in H_{2}(M ; \mathbb{Z})$. Although it is not necessary, just for aesthetical reasons we can suppose without loss of generality that $\eta_{C}$ is the unique harmonic representative of $\left[\eta_{C}\right]$ hence both $\eta_{C}$ and $J_{C}=* \eta_{C}$ are closed that is, represent cohomology classes within $H^{2}(M)$.

Note that, regardless what the symmetry $C$ actually is, in order $Q_{C,[\Sigma]}$ not to be trivial i.e., $Q_{C,[\Sigma]} \neq 0$, we need $[0] \neq[\Sigma] \in H_{2}(M ; \mathbb{Z})$ as well as $[0] \neq\left[* J_{C}\right] \in H^{2}(M)$. Both conditions are met if we demand $M$ to satisfy the topological condition that the free part of its second singular homology group $H_{2}(M ; \mathbb{Z})_{\text {free }} \cong \mathbb{Z}^{\mathrm{rk} H_{2}(M ; \mathbb{Z})}$ be non-zero (i.e., the rank of $\mathrm{H}_{2}(M ; \mathbb{Z})$ be non-zero). Moreover, at this level of generality all cohomology classes in $H^{2}(M)$ are premitted to play the role of $\left[* J_{C}\right\rfloor$ consequently the number of linearly independent secondary Noether currents is equal to $b^{2}(M)$. Therefore if $\operatorname{rk} H_{2}(M ; \mathbb{Z})>1$ that is, $b^{2}(M)>1$ then we have "too many" options to introduce non-trivial secondary 
Noether charges for a given symmetry. Consequently, the optimal situation for this secondary theory is when $\operatorname{rk} H_{2}(M ; \mathbb{Z})=1$ (at this level of generality).

To summarize, we have proved.

Lemma 3.1 Let $(M, g)$ be a (non-)compact oriented (pseudo-)Riemannian 4-manifold satisfying the topological condition $\mathrm{H}_{2}(M ; \mathbb{Z})_{\text {free }} \not\{\{0\}$. Let moreover a classical relativistic field theory be given over $(M, g)$ defined by its Lagrangian density $L(\Phi, g) \in \Omega^{4}(M)$. Assume that $C: \mathbb{R} \rightarrow \mathscr{M}$ is a symmetry of the theory such that the corresponding total derivative $\theta_{C} \in \Omega^{3}(M)$ satisfying the gauge fixing condition $\mathrm{d}^{*} \theta_{C}=0$ is closed (hence in fact $\theta_{C}=0$ ).

Then there exist a 2-form $0 \neq J_{C} \in \Omega^{2}(M)$ representing a non-trivial de Rham cohomology class $0 \neq\left[* J_{C}\right] \in H^{2}(M)$ as well as a closed oriented surface $\Sigma \subset M$ representing a non-trivial singular homology class $[0] \neq[\Sigma] \in H_{2}(M ; \mathbb{Z})_{\text {free }}$ such that the associated quantity $Q_{C, \Sigma}:=\int_{\Sigma} * J_{C} \in \mathbb{R}$ is not zero and depends only on $\left[* J_{C}\right] \in H^{2}(M)$ and $[\Sigma] \in H_{2}(M ; \mathbb{Z})_{\text {free }}$. We denote this quantity by $Q_{C,[\Sigma]}$ and call the secondary or topological Noether charge associated with the symmetry $C$ and the classes $\left[* J_{C}\right\rfloor$ and $[\Sigma]$. For a given symmetry $C$ the number of linearly independent cohomology classes $\left[* J_{C}\right]$ is equal to $\mathrm{rk} \mathrm{H}_{2}(M ; \mathbb{Z})$.

Note that what we have done is in fact simple: We interpret the a priori existing cohomology classes of $M$ as certain physical quantities whenever a theory $L(\Phi, g)$, possessing certain type of symmetries, has been formulated over $M$.

Let us apply this theory for diffeomorphisms in pure gravity in four dimensions. Let $\omega_{M} \in \Omega^{4}(M)$ defined by $\omega_{M}:=* 1$ be the volume form of $(M, g)$ and take the usual Einstein-Hilbert Lagrangian $L_{E H}(g):=\left(\operatorname{Scal}_{g}-2 \Lambda\right) \omega_{M}$ with cosmological constant $\Lambda \in \mathbb{R}$ and consider its variation with respect to a 1-parameter subgroup $\left\{f_{t}\right\}_{t \in \mathbb{R}}$ of the orientation-preserving diffeomorphism group $\operatorname{Diff}^{+}(M)$ of the underlying space-time manifold $M$ while the metric $g$ is kept fixed. That is we define our curve by $C(t):=f_{t}^{*} g \in \mathscr{M}$ for all $t \in \mathbb{R}$. The infinitesimal generator of this subgroup is a compactly supported vector field $X \in C_{c}^{\infty}(M ; T M)$. Since a diffeomorphism acts on $k$-forms via pullback and the scalar curvature is invariant under diffeomorphisms, $L_{E H}(C(t))=f_{t}^{*} L_{E H}\left(f_{t}^{*} g\right)=f_{t}^{*} L_{E H}(g)$ hence the corresponding infinitesimal variation takes the shape

$$
\delta_{C} L_{E H}(g)=\lim _{t \rightarrow 0} \frac{1}{t}\left(1-f_{t}^{*}\right) L_{E H}(g)=L_{X}\left(L_{E H}(g)\right)
$$

where $L_{X}$ denotes the Lie derivative with respect to $X$. Substituting the Lagrangian and applying Cartan's formula we thus get

$$
\begin{aligned}
\delta_{C} L_{E H}(g) & =l_{X}\left(\mathrm{~d}\left(\left(\operatorname{Scal}_{g}-2 \Lambda\right) \omega_{M}\right)\right)+\mathrm{d}\left(l_{X}\left(\left(\operatorname{Scal}_{g}-2 \Lambda\right) \omega_{M}\right)\right) \\
& =\mathrm{d}\left(\left(\operatorname{Scal}_{g}-2 \Lambda\right) l_{X} \omega_{M}\right) .
\end{aligned}
$$

While $\delta_{C} L_{E H}(g) \neq 0$ in general, nevertheless we find that $\delta_{C} S_{E H}(g)=\int_{M} \mathrm{~d}\left(\left(\mathrm{Scal}_{g}-2 \Lambda\right) l_{X} \omega_{M}\right)=0$ by Stokes' theorem hence the Einstein-Hilbert action itself is invariant consequently diffeomorphisms are both off or on shell symmetries of general relativity with possibly non-vanishing cosmological constant. The associated total derivative up to an exact term looks like $\theta_{C}=\left(\mathrm{Scal}_{g}-2 \Lambda\right) t_{X} \omega_{M}$ in some gauge probably not satisfying the condition $\mathrm{d}^{*} \theta_{C}=0$. 
In order not to get lost in the gauge fixing problem assume instead that (i) we are on shell i.e., Einstein's equation $\operatorname{Ric}_{g}=\Lambda g$ is valid hence $\theta_{C}=2 \Lambda l_{X} \omega_{M}$ and (ii) the cosmological constant vanishes yielding $\theta_{C}=0$. Consequently the diffeomorphism symmetry in on shell pure gravity with vanishing cosmological constant has vanishing associated (primary) Noether charge. However substituting $\theta_{C}=0$ into (3) (and referring to the Hodge decomposition theorem) we get $\mathrm{d}^{*} \varphi_{C}=0$ and $\mathrm{d} \eta_{C}=0$ consequently in this physically important situation we can interpret the cohomology classes $\left[\eta_{C}\right] \in H^{2}(M)$ as Hodge duals of currents $J_{C}$ in secondary Noether theory.

As we stressed even in the formulation of Lemma 3.1, interesting secondary Noether theory emerges only if the underlying manifold is topologically non-trivial in the sense formulated there. At this point, by taking e.g. a survey on known solutions (Stephani et al. 2003), we make an observation which is completely independent of our considerations taken so far-hence in our opinion is very interesting!-namely: Apparently all explicitly known 4 dimensional black hole solutions in vacuum general relativity with vanishing cosmological constant satisfy the topological condition formulated in Lemma 3.1. This intuitively means that because of some general reason a black hole is even topologically recognizable as a two dimensional "hole" in space-time. In fact with an appropriate restriction this observation can be proved (Etesi 1998) and can be considered as a global topological counterpart of well-known black hole uniqueness theorems (Heusler 1996). In accordance with this provable version (Etesi 1998) we suppose from now on that: $(M, g)$ is a 4 dimensional solution of the Einstein's equation $\operatorname{Ric}_{g}=0$ and describes a single stationary asymptotically flat black hole; hence $\operatorname{rk} H_{2}(M ; \mathbb{Z})=1$ (which apparently corresponds to the case that a "single" black hole is present). In this case the homology class of the "instantaneous" event horizon of the black hole as an (immersed) surface $i: \Sigma \leftrightarrow M$ represents a non-zero element $[\Sigma] \in H_{2}(M ; \mathbb{Z})_{\text {free }} \cong \mathbb{Z}$.

Then we proceed as follows: Like the original volume form $* 1=*_{g} 1=\omega_{M} \in \Omega^{4}(M)$, the induced area form $*_{i^{*} g} 1=: \omega_{\Sigma} \in \Omega^{2}(\Sigma)$ of the "instantaneous" event horizon $\Sigma$ is closed consequently it represents a class $\left[\omega_{\Sigma}\right] \in H^{2}(\Sigma)$ which is not zero since the event horizon has finite area. Then exploiting singleness and stationarity, the "instantaneous" event horizon $\Sigma$ is connected and its area $\operatorname{Area}_{g}(\Sigma)=\int_{\Sigma} \omega_{\Sigma}$ is constant in time consequently we can suppose that the area form is proportional to the Hodge dual of the secondary Noether current with a time-independent constant. In other words with any choice for the Hodge dual of the secondary Noether current $J_{C} \in \Omega^{2}(M)$ the $\omega_{\Sigma}$ satisfies that Const $\left[i^{*}\left(* J_{C}\right)\right]=\left[\omega_{\Sigma}\right] \in H^{2}(\Sigma) \cong \mathbb{R}$ therefore

$$
\begin{aligned}
\text { Entropy of the black hole in }(M, g) & =\text { Const }\left(\operatorname{Area}_{g}(\Sigma)\right)=\text { Const } \int_{\Sigma} \omega_{\Sigma}=\text { Const } \int_{\Sigma} * J_{C} \\
& =\text { Const } Q_{C,[\Sigma]}
\end{aligned}
$$

offering a natural way to normalize $Q_{C,[\Sigma]}$ to be equal to the entropy of the black hole i.e.

$$
\text { Entropy of the black hole in }(M, g)=Q_{C,[\Sigma]} \text {. }
$$

Accepting this choice of normalization therefore a natural physical interpretation of this abstract conserved quantity also emerges, namely: If a 4 dimensional space-time $(M, g)$ is a solution of the vacuum Einstein's equation with vanishing cosmological constant and describes a single stationary asymptotically flat black hole then the secondary Noether charge associated to an orientation-preserving diffeomorphism of the space-time manifold 
is not zero and as a secondary conserved quantity is equal to the entropy of the black hole (cf. Wald 1993).

We are now in a position to make our crucial observation: Both the previously introduced set-theoretic and the black hole entropy here give rise to conserved quantities assigned to one and the same process namely the permutation of the points of a given differentiable manifold by diffeomorphisms. This manifests itself in their common diffeomorphism invariance. More precisely: (2) as the set-theoretic entropy of the abstract set $X$ underlying the black hole space-time $M$ is invariant in the formal sense while (4) as the black hole entropy of $(M, g)$ is invariant in the secondary Noether sense. Hence these two scalars are expected to obey a functional relation like

\section{$F($ Entropy of the black hole in $(M, g)$, Entropy of $X$ in its manifold-macrostate $M)=0$}

and accepting the validity of the implicit function theorem then at least locally the black hole entropy can be recovered from this as follows:

Entropy of the black hole in $(M, g)=f($ Entropy of $X$ in its manifold-macrostate $M)$.

Both quantities express entropy hence are subject to positivity, subadditivity, etc. moreover (4) depends on both the smooth structure on $M$ and a metric $g$ on it while (2) depends only on the smooth structure on $M$. These properties force the two entropy expressions in fact to be proportional. Therefore the (stationary, asymptotically flat, single) black hole entropy (4) factorizes like

Entropy of the black hole in $(M, g)=$ Const $_{g} \cdot($ Entropy of $X$ in its manifold-macrostate $M)$

consisting of a metric term Const ${ }_{g}>0$ and the pure set-theoretic entropy term from (2) of the underlying manifold continuum. We can even generalize this "formula" for any solution of Einstein's equation by setting the left hand side to be zero if $(M, g)$ does not describe a black hole and putting Const ${ }_{g}=0$ on the right hand side, too.

The time has come to complete the circle of our arguments. On the mathematical side, the formal concept of the arithmetical continuum (or the set $\mathbb{R}$ of real numbers) contains an inherent uncertainty or fuzziness in the sense that the effective identification of the arithmetical continuum with its individual disjoint constituents, the points, cannot be carried out (our interpretation of Theorem 2.1). On the physical side, the nowadays accepted formal mathematical description of our intuitive concept of the spatial or temporal continuum in terms of the arithmetical continuum lifts the purely formal-and concerning its origin, metaphysical-concept, the same point again, to an ontological level. We may then ask ourselves whether or not this sort of description of space-time in a mathematical model of a physical theory introduces a similar uncertainty or fuzziness into the physical theory. Let us formulate our question more carefully. In the modern understanding by a physical theory one means a two-level description of a certain class of natural phenomena: The theory possesses a syntax provided by its mathematical core structure and a semantics which is the meaning i.e. interpretation of the bare mathematical model in terms of physical concepts. Consider a physical theory whose semantics contains a description of space and time (like general relativity) and its syntax uses the arithmetical continuum to mathematically describe the thing which corresponds to the space-time continuum at the semantical level of the theory (like general relativity). Then we may ask whether or not the uncertainty or fuzziness at the syntactical level of the physical theory (introduced by the utilization of the arithmetical continuum) shows up at the semantical level of the physical theory as well? To answer this for a given physical theory, we have to search among its physical concepts 
which describe uncertainty, fuzziness, or disorder at the semantical level and check their counterparts at the syntactical level. Of course the basic physical concept of this kind is the entropy.

Therefore, in this context, one can be concerned whether or not entropy within classical general relativity, appearing in its semantics in the form of black hole entropy (Bardeen et al. 1973; Hawking 1971), simply comes from its syntax i.e. has a pure mathematical origin only (hence probably not corresponding to any "objective" thing in the world)? This suspicion is also supported in some extent by the several controversial (geometrical, thermodynamical, quantum and information theoretic, etc.) properties of the one and the same black hole entropy. Our analysis of the black hole entropy formula in general relativity culminating in its formal factorization (5) into the product of a metric term and a pure set-theoretic term points at least in part towards a set-theoretic origin. That is, even if the metric term in (5) indeed corresponds to the "physical part" of black hole entropy, the next term could be a "pure mathematical" or more precisely a "pure set-theoretic" contribution only. This could be an example of how certain physical statements within the physical theory of general relativity are "contaminated" by the underlying mathematical model akin to the situation in quantum field theory (Baez 2016).

However if one prefers one can read (5) in two extreme ways as well, going into exactly the opposite directions as follows. The first is that black hole entropy is of pure mathematical origin without any physical content hence e.g. the long-sought as well as quite problematic physical degrees of freedom responsible for black hole entropy (far from being complete cf. e.g. Strominger and Vafa 1996) would in fact be not physical at all but would simply coincide with the purely "mathematical degrees of freedom" of the point constituents of the arithmetical continuum used to formulate general relativity mathematically. We have to acknowledge that as long as the physical origin of black hole entropy is not confirmed by the experimental discovery of e.g. black hole radiation (Hawking 1975; Wald 1975 ) or other thermal phenomena, this possibility cannot be a priori refuted. The second extreme reading is that black hole entropy is of pure physical origin without any mathematical content which arises if one rather wishes to accept the physical origin of black hole entropy (and e.g. looks forward its experimental discovery). Then (5) can be interpreted as an argument for the "physical origin" of what we have called the inherent fuzziness or uncertainty within the set of real numbers. This interpretation then could explain the expected independence of the set-theoretic entropy (2) of any axiomatic system $S$ which is in accordance with the universal character of Theorem 2.1.

Finally, in the realm of the latter extreme interpretation, for future investigation we record here another consequence of the entropy formulas (2) and (4), too. Let $M$ be a differentiable 4-manifold with $H_{2}(M ; \mathbb{Z})_{\text {free }} \cong \mathbb{Z}$ and equipped with a metric $g$. Suppose $\Sigma \subset M$ is a homologically non-trivial closed minimal surface (mathematical equivalent of the apparent horizon of a single black hole). Consider the hypothetical measure $\Gamma_{S}$ on the group $\operatorname{Bij}(X)$ of all set-theoretic bijections of $M$ as a set $X$. Although we expect $\Gamma_{S}(\operatorname{Bij}(X))=+\infty$ to hold, by the aid of (5) the measure of the subgroup $\operatorname{Diff}(M) \varsubsetneqq \operatorname{Bij}(X)$ of $\operatorname{diffeomorphisms}$ formally looks like

$$
\Gamma_{S}(\operatorname{Diff}(M))=\mathrm{e}^{\frac{\text { Areag }_{g}(\mathcal{L})}{\text { Const }_{S} \text { Constg }}}<+\infty
$$

where $0<$ Const $_{S}<+\infty$ is the constant depending on the axiomatic system $S$ used to define $\Gamma_{S}$ while $0<$ Const $_{g}<+\infty$ is the constant depending only on the metric. 
Acknowledgements Open access funding provided by Budapest University of Technology and Economics (BME). The author is grateful to the Referee for the valuable comments on the earlier version of the manuscript.

Open Access This article is distributed under the terms of the Creative Commons Attribution 4.0 International License (http://creativecommons.org/licenses/by/4.0/), which permits unrestricted use, distribution, and reproduction in any medium, provided you give appropriate credit to the original author(s) and the source, provide a link to the Creative Commons license, and indicate if changes were made.

\section{References}

Baez, J. (2016). Struggles with the continuum. arXiv:1609.0142 [math-ph] (preprint), 42 pp.

Bardeen, J. M., Carter, B., \& Hawking, S. W. (1973). The four laws of black hole mechanics. Communications in Mathematical Physics, 31, 161-170.

Chaitin, G. J. (1992). Algorithmic information theory. Cambridge: Cambridge University Press.

Chern, S.-S., \& Simons, J. (1974). Characteristic forms and geometric invariants. Annals of Mathematics, 99, 48-69.

Dolan, B. P. (2018). A tale of two derivatives: Phase space symmetries and Noether charges in diffeomorphism invariant theories. Physical Review D, 98, 044009.

Etesi, G. (1998). A global uniqueness theorem for stationary black holes. Communications in Mathematical Physics, 195, 691-697.

Geroch, R. P., \& Hartle, J. (1986). Computability and physical theories. Foundations of Physics, 16, 533-550.

Hawking, S. W. (1971). Gravitational radiation from colliding black holes. Physical Review Letters, 26, 1344-1346.

Hawking, S. W. (1975). Particle creation by black holes. Communications in Mathematical Physics, 43, 199-220.

Heusler, M. (1996). Black hole uniqueness theorems. Cambridge Lecture Notes in Physics (Vol. 6). Cambridge: Cambridge University Press.

Russell, B. (1910). The problems of philosophy. London: Williams and Norgate VIII.

Schlesinger, K.-G. (2014a). Entropy, heat and Gödel incompleteness. arXiv:1404.7433 [physics.gen-ph] (preprint), $7 \mathrm{pp}$.

Schlesinger, K.-G. (2014b). Notes on the firewall paradox, complexity and quantum theory. arXiv:1412.3146 [physics.gen-ph] (preprint), $31 \mathrm{pp}$.

Stephani, H., Kramer, D., MacCallum, M., Hoenselaers, C., \& Herlt, E. (2003). Exact solutions of Einstein's field equations. Cambridge: Cambridge University Press.

Strominger, A., \& Vafa, C. (1996). Microscopic origin of the Bekenstein-Hawking entropy. Physics Letters $B, 379,99-104$.

Wald, R. M. (1975). On particle creation by black holes. Communications in Mathematical Physics, 45, 9-45.

Wald, R. M. (1993). Black hole entropy is Noether charge. Physical Review D, 48, 3427-3431.

Weyl, H. (1994). The continuum. New York: Dover Publications, Inc.

Publisher's Note Springer Nature remains neutral with regard to jurisdictional claims in published maps and institutional affiliations.

Gábor Etesi is a Hungarian mathematical physicist graduated from the Eötvös Roland University Budapest, Hungary in 1995. Currently he is a reader (associate professor) of geometry at the Department of Geometry, Mathematical Institute of the Budapest University of Technology and Economics. His main research field is mathematical problems of classical and quantum Yang--Mills theory and general relativity. For further information visit his homepage http://www.math.bme.hu/ etesi. 